\title{
Phase Stability of Concentrated Dairy Products
}

\author{
Erik ten Grotenhuis, ${ }^{*}$ Remco Tuinier, ${ }^{*}, \dagger$ and Cornelus G. de Kruif, ${ }^{*}, \dagger$ \\ ${ }^{*}$ NIZO Food Research, P. O. Box 20, 6710 BA Ede, The Netherlands \\ †Van 't Hoff Laboratory for Physical and Colloid Chemistry, Utrecht University, P. O. Box 80.051, 3508 TB Utrecht, \\ The Netherlands
}

\begin{abstract}
The interactions between the two most important colloids in milk, fat globules and casein micelles, were investigated. Mixtures of oil droplets (as a model for fat globules) and casein micelles were prepared, and their phase behavior was studied. It was found that the oil droplets and the casein micelles phase separate as a result of depletion interaction. The experimentally determined phase boundary is consistent with a prediction by theory that involves no adjustable parameters. Furthermore, it is shown that the kinetics of phase separation can be explained by the differences in viscosity of the samples. The results are of relevance to the behavior of concentrated dairy products such as whipping cream and evaporated milk.
\end{abstract}

(Key words: casein micelle, emulsion, phase behavior)

Abbreviation key: MSX = Mastersizer X, SMUF = simulated milk ultrafiltrate.

\section{INTRODUCTION}

Milk is a prime example of a natural, but mixed, colloidal system. The fat globules and the casein micelles are the colloids that give milk its white appearance. Various dairy products are made by processing milk in different ways. Cream is produced by centrifugation of milk and is a product that is relatively concentrated in fat globules, but reduced in casein micelle concentration. Evaporation leads to increased concentrations of both the casein micelles and the fat globules. In Table 1, the approximate fat and casein contents of these products are compared (Snoeren et al, 1984; Walstra and Jenness, 1984; Jeurnink and de Kruif, 1993). The calculation of the volume fractions of casein micelles in these products was made as described for a solution of skim milk powder and is explained in the Materials and Methods section of this paper. An in-

Received March 13, 2002.

Accepted September 9, 2002. NL.

Corresponding author: C. G. de Kruif; e-mail: DEKRUIF@NIZO. crease in concentrations of the colloids in the milk changes the thermodynamic properties of the suspension. This could be the cause of problems related to gelling of evaporated milk and phase separation in cream. The reasons for the occurrence of these problems are not completely clear, but it is likely that other factors such as chemical reactions are of at least equal importance. Years of experience taught processors how the processing should be done in order to prevent these instability problems, but a good theoretical understanding is still lacking.

Many studies are available that describe factors involving the stability of emulsions (Dalgleish, 1997; Dickinson, 1997; Robins, 2000). Aspects considered are the use of milk proteins that can stabilize the surface, or interface, of oil droplets (Dickinson, 1997), but also the physical processes that cause emulsions to become unstable, i.e., creaming, flocculation, and coalescence. Furthermore, analytical techniques have been discussed with relevance to measuring creaming of emulsion droplets (Robins, 2000). Casein micelles have also been the subject of many studies. Aspects investigated include their detailed structure (Dalgleish, 1998), their size distribution (Holt, 1985), and the interactions between casein micelles (Holt and Horne, 1996; Horne, 1998; De Kruif, 1999), explaining the renneting and the acidification processes that are commonly used to produce cheese and yoghurt. Although much is known about emulsions and casein micelles, only limited literature is available on the interactions of emulsion droplets and caseins. Xu et al. (1998) reported a stabilizing effect on emulsions by the addition of small sodium caseinate micelles (confusingly labeled as submicelles by $\mathrm{Xu}$; these should not be interpreted as the much discussed building blocks of casein micelles). The proposed mechanism is that the (sub)micelles prevent the emulsion droplets from approaching each other, because they form a layer around the droplets. Dickinson and Golding (1997), by studying the creaming behavior of emulsions with different amounts of sodium caseinate, demonstrated that sodium caseinate (sub)micelles can cause depletion flocculation of emulsion droplets. Light microscopy and rheological measurements supported the depletion interaction mechanism. The 
Table 1. Fat and casein content of several dairy products.

\begin{tabular}{lll}
\hline Product & $\begin{array}{l}\text { Fat content } \\
\% \text { wt/wt } \\
\text { (volume fraction) }\end{array}$ & $\begin{array}{l}\text { Casein micelle content } \\
\% \text { wt/wt } \\
\text { (volume fraction) }\end{array}$ \\
\hline Milk & $\begin{array}{l}3.9(0.043) \\
\text { Cream }\end{array}$ & $\begin{array}{l}2.6(0.106) \\
1.8(0.108)\end{array}$ \\
Evaporated Milk & $10(0.11)$ & $6(0.26)$ \\
\hline
\end{tabular}

change in the flow behavior when milk is concentrated has been attributed to the increase in solids content, as well as to the formation of complexes between fat globules and proteins (Vélez-Ruiz and Barbosa-Cánovas, 2000). There is no report of the nature of the interaction between casein micelles (which are much larger than caseinate (sub)micelles) and emulsion droplets or fat globules.

In this paper, a study is presented on the phase behavior of casein micelles and oil droplets. The phase behavior of these colloids is explained by depletion interaction theory. Oil droplets were chosen as a model system for fat globules to eliminate any possible effects of fat crystallisation.

\section{MATERIALS AND METHODS}

\section{Materials}

Sunflower oil (brandname: Reddy) was purchased from a local supermarket. The density of the oil was $0.9204 \mathrm{~kg} / \mathrm{L}$. Sodium caseinate was obtained from DMV International (Veghel, The Netherlands). It contained a minimum protein content of $88 \%$, wt/wt, and had a maximum fat content of $6 \%$, wt/wt. As a model for the serum phase of milk, i.e., milk without proteins and fats simulated milk ultrafiltrate (SMUF) was used (Jenness and Koops, 1962). It is prepared by making a solution in double-distilled water containing $0.158 \% \mathrm{KH}_{2} \mathrm{PO}_{4}$, $0.120 \% \quad \mathrm{~K}_{3}$ Citr. $\mathrm{H}_{2} \mathrm{O}, 0.179 \% \quad \mathrm{Na}_{3}$ Citr. $2 \mathrm{H}_{2} \mathrm{O}, 0.018 \%$ $\mathrm{K}_{2} \mathrm{SO}_{4}, \quad 0.132 \% \quad \mathrm{CaCl}_{2} \cdot 2 \mathrm{H}_{2} \mathrm{O}, \quad 0.065 \% \quad \mathrm{MgCl}_{2} \cdot 6 \mathrm{H}_{2} \mathrm{O}$, $0.030 \% \mathrm{~K}_{2} \mathrm{CO}_{3}$, and $0.060 \% \mathrm{KCl}$ (all \% wt/wt). Low-heat skim-milk powder (Nilac) was produced at the NIZO food research pilot plant by spray drying. Nilac has a casein content of $27 \%$. As a preservative, thiomersal was added to the model emulsions at a concentration of $0.02 \%$.

\section{Methods}

Sodium caseinate $(2.6 \%)$ was dissolved in SMUF. After complete dissolution, oil was mixed with this solution in a 1:1 ratio. First, a coarse emulsion was prepared by using an Ultra Turrax (Polytron, $\mathrm{CH}$ ) mixer and mixing for $1 \mathrm{~min}$. This was immediately followed by homogenization of the emulsion in a Rannie 8.30 labo-

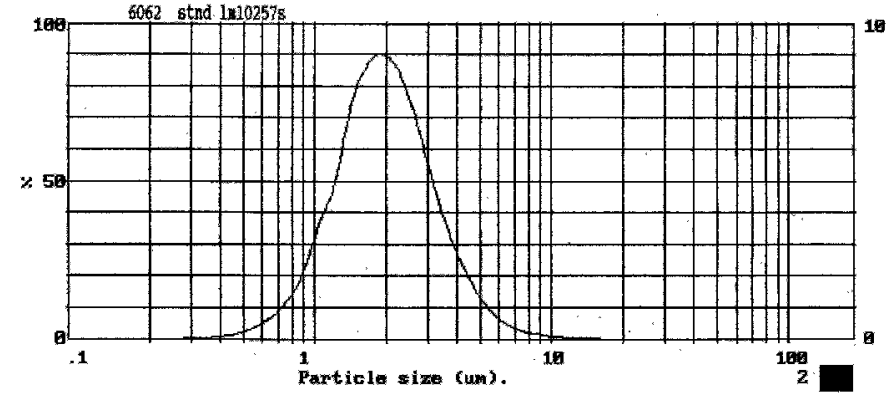

Figure 1. Oil droplet size distribution of 50\% w/w oil emulsion measured with a Malvern Mastersizer X (Malvern Instruments; www.malverninstruments.com).

ratory homogenizer. The emulsions were passed 10 times at 100 bar. Thiomersal was added, and the emulsions were stored at $4^{\circ} \mathrm{C}$ until usage.

Nilac (46.15 g) was dissolved in $100 \mathrm{ml}$ of SMUF, yielding a dispersion with a casein micelle volume fraction of 0.5 [46.15 g of Nilac contains approx. $14.8 \mathrm{~g}$ of casein, resulting in a volume of $57.6 \mathrm{ml}$ (Snoeren et al., 1984); the remaining volume of water is then approximately $100-42.8=57.2 \mathrm{ml}$; hence a volume fraction of casein micelles of about 0.5 ). If required, even larger amounts of Nilac were dissolved.

The size distribution of the emulsion droplets was measured with a Malvern Mastersizer X (MSX; Malvern; www.malverninstruments.com) static light-scattering apparatus. The diffraction of laser light by a diluted portion of the emulsion is detected on an array of detectors and transformed into a size distribution by the software of the MSX.

A Turbiscan MA 1000 was used to measure the stability of the emulsions. The turbidity of the emulsion is measured as a function of the height of the emulsion in a vertical tube with an inner diameter of $13 \mathrm{~mm}$. The light that is reflected by the emulsion increases with the oil droplet concentration locally present in the emulsion. With the Turbiscan the distribution of the phases of the emulsions can be determined quite accurately, since the resolution of the apparatus is close to $0.1 \mathrm{~mm}$. Furthermore, the measurements can be performed as a function of time. This allows the monitoring of the time-dependent behavior of the emulsions. Typical behaviors that can be observed are creaming, which appears as a gradual change of the turbidity as a function of height, and phase separation that is recognized by a clear and sharp interface between the fat-rich and the casein-rich phase.

\section{RESULTS}

The size distribution of the emulsion droplets is given in Figure 1. The volume-surface weighted average di- 
ameter $(\mathrm{d} 3 ; 2)$ of the droplets was $1.7 \mu \mathrm{m}$. The polydispersity of the emulsion droplets, defined as the standard deviation normalized by the average diameter, was $35 \%$. Upon storage at $4^{\circ} \mathrm{C}$, the emulsion was stable for at least 3 wk. Dickinson and Golding (1997) showed that an amount of $2 \%$ sodium caseinate was sufficient for complete coverage of the emulsion droplets, but that higher concentrations could lead to depletion flocculation. Dickinson and Golding used this amount to stabilize $35 \%$ oil emulsions. In this paper, an amount of $2.6 \%$ of sodium caseinate was used to stabilize a $50 \%$ oil emulsion. Such an amount is less than used by Dickinson and Golding in relation to the concentration of oil. Therefore, it is not expected that any stability problems caused by depletion flocculation due to an excess of caseinate would occur. The particle size was checked periodically and had remained unchanged.

Several samples containing different quantities of oil droplets and casein micelles (expressed as volume fraction) were prepared and stored in Turbiscan tubes. These were measured at regular intervals increasing from $1 \mathrm{~h}$ directly after preparation to a few days after $3 \mathrm{wk}$ of storage. The samples were stored at either 4 or $20^{\circ} \mathrm{C}$ and were monitored both visually and with the Turbiscan apparatus. By eye, it was almost impossible to detect creaming or phase separation at an early stage, because both the phase rich in emulsion droplets and the phase rich in casein micelles were white. However, with the Turbiscan, the difference between a homogeneous and a creamed or phase-separated sample can be detected at an early stage. By checking the samples at regular intervals, it was determined which of the samples phase separated and which remained stable. The phase diagrams for the mixtures of oil droplets and casein micelles are presented in Figures 2 and 3. Phase separation between oil droplets and casein micelles occurs at volume fractions of both components above the phase boundary. The experimentally determined phase boundary is equal for samples stored at $4^{\circ} \mathrm{C}$ and at $20^{\circ} \mathrm{C}$.

In Figure 4, three creaming profiles are compared. These were recorded for samples stored at $20^{\circ} \mathrm{C}$ and with a fat volume fraction of 0.108 . The samples had different volume fractions of casein micelles: $0.03,0.33$, and 0.45 . The first sample is below the phase boundary and remains stable; the other two will phase separate. It can be observed that the sample with the low concentration of casein micelles is not stable after $30 \mathrm{~h}$. After this time, the sample displays natural creaming of the oil droplets. The final position of the interface of this sample is higher than for the samples with a high volume fraction of casein micelles. The position of the interface decreases with increasing volume fraction of casein micelles. At high casein micelle concentrations,

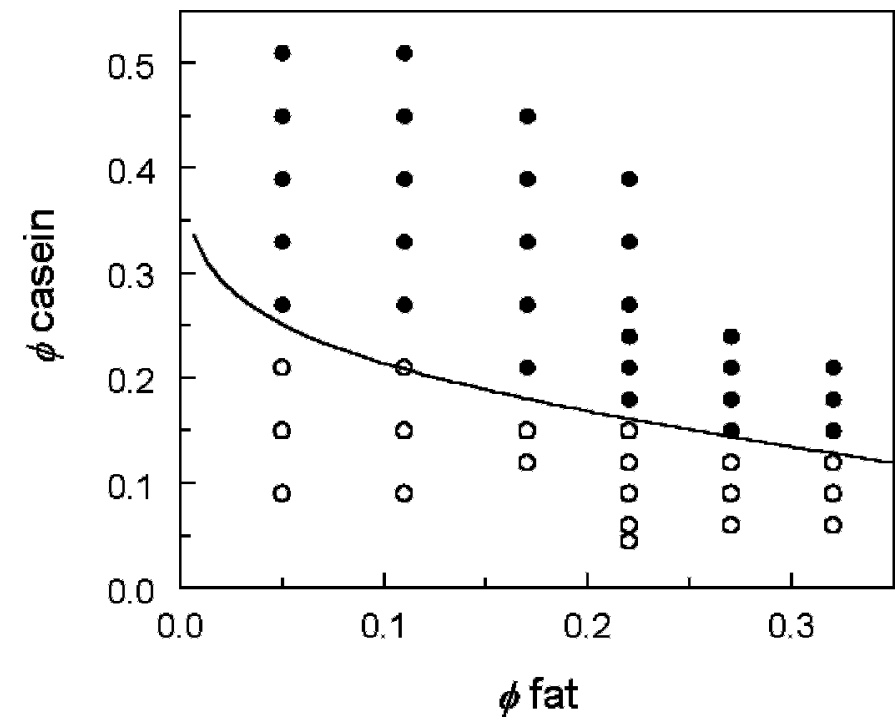

Figure 2. Phase diagram with experimental phase boundary determined with a Turbiscan for samples stored at $20^{\circ} \mathrm{C}$. $\bigcirc$ stable phase separated curve is drawn to guide the eye.

the upper phase is less concentrated in oil droplets. A similar graph has been plotted for samples with identical volume fractions of casein micelles of 0.36 , but with different volume fractions of oil: $0.108,0.163$, and 0.217 (Figure 5). As can be expected, the oil droplet phase has the largest volume for the sample with the highest volume fraction of oil droplets. The samples start to phase separate immediately after mixing the oil droplets and the casein micelles. Initially, the process of

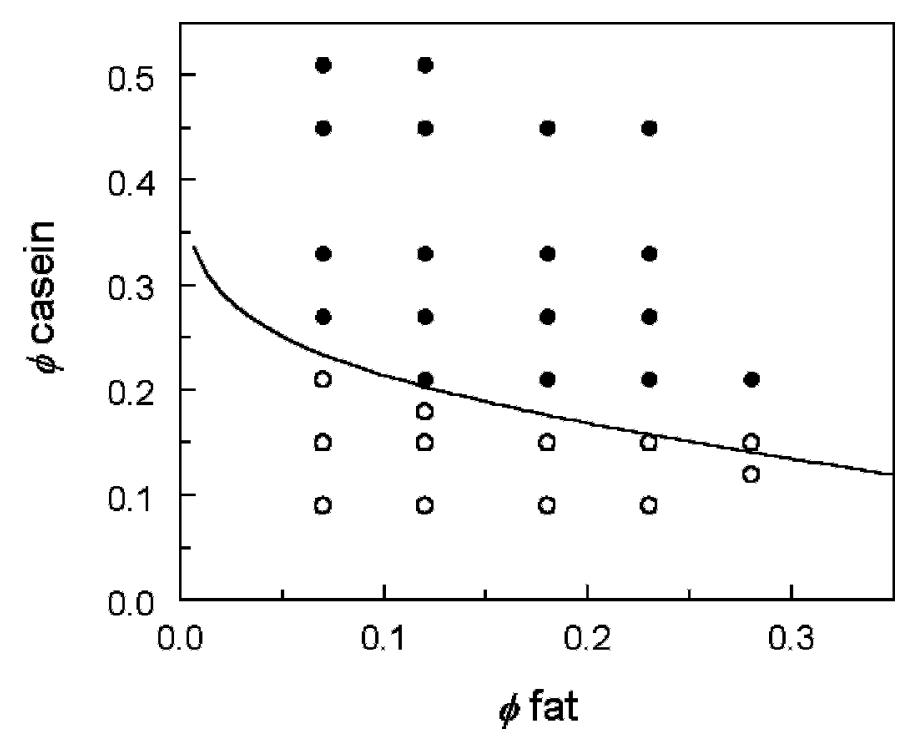

Figure 3. Phase diagram with experimental phase boundary determined with a Turbiscan for samples stored at $4^{\circ} \mathrm{C}$. $\bigcirc$ stable phase separated curve is drawn to guide the eye. 


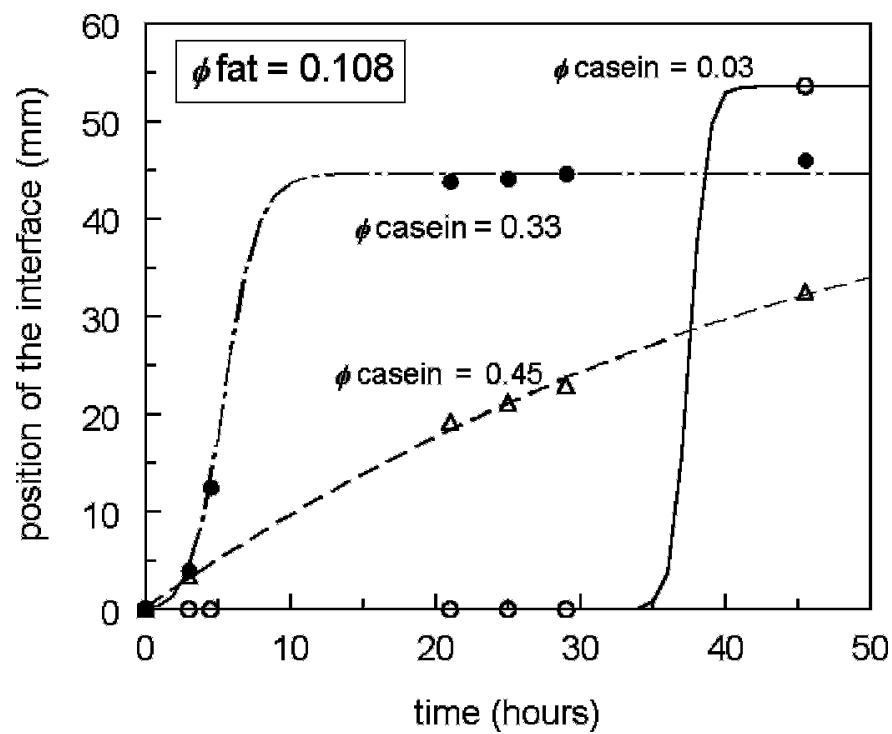

Figure 4. Creaming profiles of samples with equal volume fractions of oil droplets, but different volume fractions of casein micelles. $\bigcirc \phi$ casein $0.03 \bullet \phi$ casein $0.33 \Delta \phi$ casein 0.45 curves are drawn to guide the eye.

phase separation progresses rapidly, but the samples are slowly reaching their final state after more than 20 $\mathrm{h}$. To check whether the kinetics of phase separation depend on the fat volume fraction, or can be scaled to a universal behavior, the final position of the interface was determined as a function of the casein micelle vol-

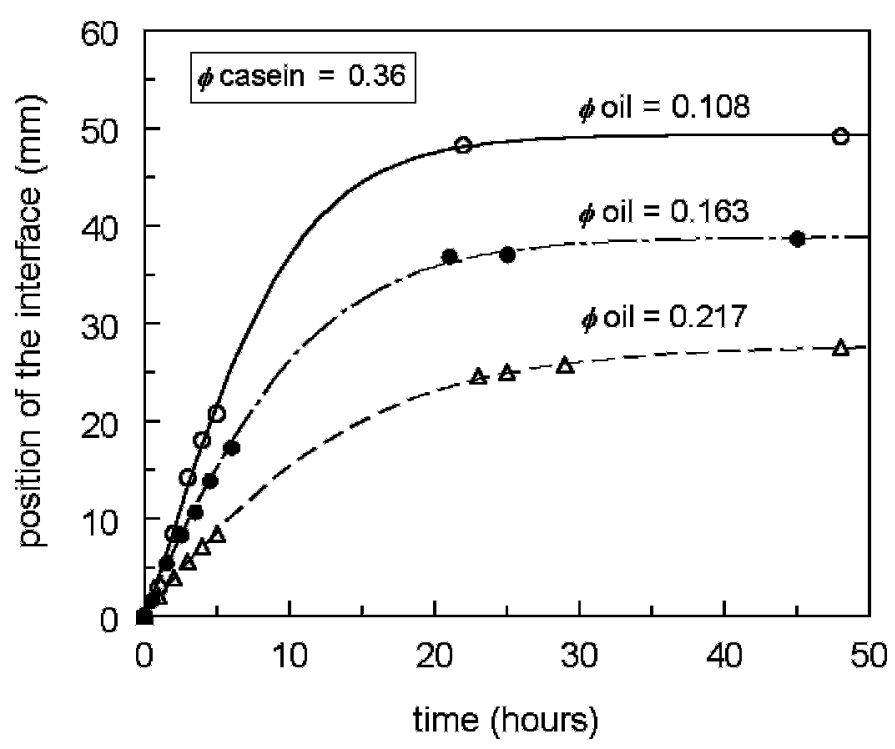

Figure 5. Creaming profiles of samples with equal volume fractions of casein micelles, but different volume fractions of oil droplets. $\bigcirc \phi$ oil $0.108 \bullet \phi$ oil $0.163 \triangle \phi$ oil 0.217 curves are drawn to guide the eye.

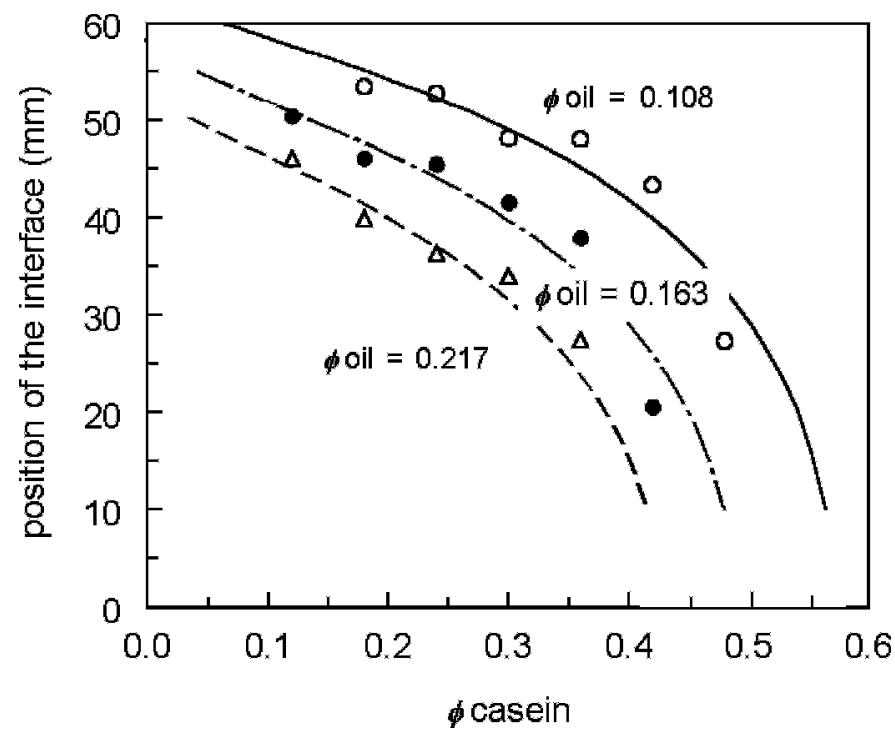

Figure 6. Position of the interface between the oil-rich upper phase and the casein-rich lower phase as a function of the volume fraction of casein micelles at three different oil droplet volume fractions. $\bigcirc \phi$ oil $0.108 \bullet \phi$ oil $0.163 \triangle \phi$ oil 0.217 curves are drawn to guide the eye.

ume fraction. In Figure 6 the curves are shown for three different volume fractions of oil droplets. It can be observed that the position of the interface decreases when the casein volume fraction increases. At every value of the casein micelle volume fraction, the interface is at a lower position for samples with a higher volume fraction of oil droplets. The relation between the position of the interface and the volume fraction of casein micelles is far from linear. At higher volume fractions of casein micelles, the position of the interface rapidly decreases. Figure 7 contains the same data as Figure 5 , but the data points have been scaled on the final position of the interface. By plotting the data in this way, the kinetics of the different samples can be compared. It can be observed that the rate at which phase separation occurs is decreased upon increasing the volume fraction of oil droplets. The data show that the samples are phase separating on a different time scale.

\section{DISCUSSION}

It was observed that casein micelles and oil droplets form stable systems at low volume fractions of both components, but tend to phase separate at higher volume fractions. The mechanism behind this behavior is thought to be depletion interaction. It is energetically favorable for both the casein micelles and the oil droplets to be surrounded by colloids of the same size. Depletion interaction is a phenomenon that has long been known to exist in mixtures of colloids and polymers 


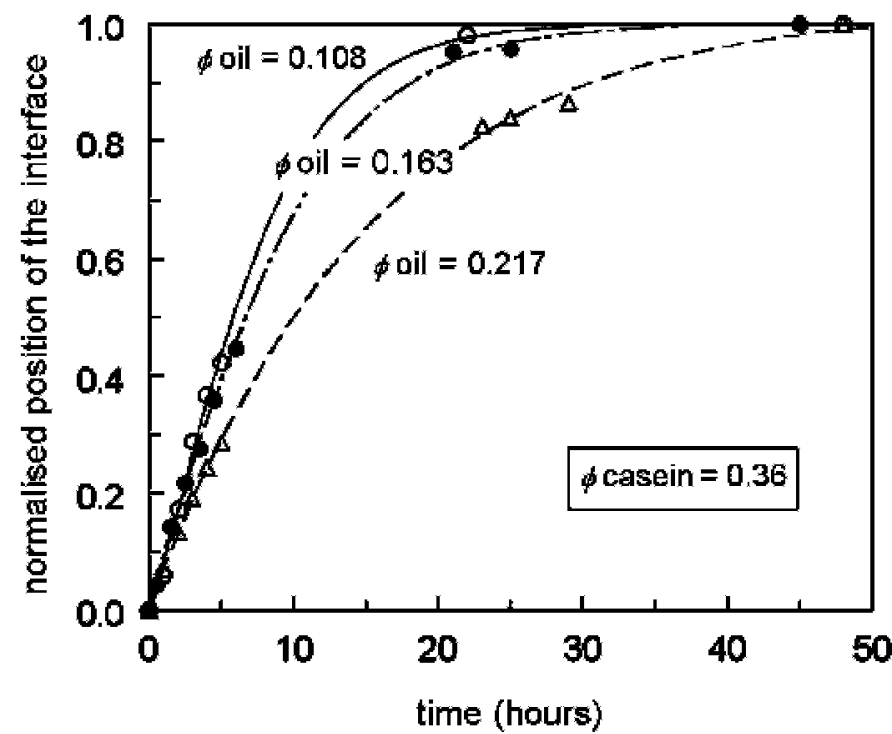

Figure 7. Creaming profiles with normalised position of the interface determined on samples with equal volume fractions of casein micelles, but different volume fractions of oil droplets. $\bigcirc \phi$ oil 0.108

- $\phi$ oil $0.163 \triangle \phi$ oil 0.217 curves are drawn to guide the eye.

(Poon, 1998). In these systems the polymers cause the colloidal particles to form a separate phase. More recently, the same effect has been shown to exist for systems consisting of colloidal particles of different sizes (Dinsmore et al., 1995). These results were supported by exact theoretical calculations (Mao et al., 1995). When the ratio between the size of large and small particles exceeds 3:1, phase separation driven by depletion interaction will occur at sufficiently high volume fractions. In the case of a mixture of casein micelles and oil droplets, the size ratio is approximately 10:1, and phase separation is expected. The depletion interaction theory (Vrij, 1976) has been applied to the system under investigation. The theory of Vrij gives a prediction of the position of the boundary where spinodal decomposition (the phase separation threshold where a system spontaneously phase separates) takes place. The theory involves no adjustable parameters. For a detailed description of the theory we refer to the paper by Tuinier et al. (1999). The position of the phase boundary depends on the overlap volume of the colloids and the (ideal) osmotic pressure of the casein solution (treated here as polymers). The osmotic pressure equals $\mathrm{c}_{\mathrm{p}} \mathrm{RT} / M$, with $\mathrm{c}_{\mathrm{p}}$ the concentration of caseins, $\mathrm{R}$ the gas constant, $T$ the temperature, and $M$ the molecular mass of the caseins. The overlap volume is a function of the size of the colloids. Only the size of the particles (diameter of oil droplets $1.7 \mu \mathrm{m}$; diameter of casein micelles $200 \mathrm{~nm}$ ) are used in the calculation. In Figure 8, the theoretical phase boundary is compared with the exper-

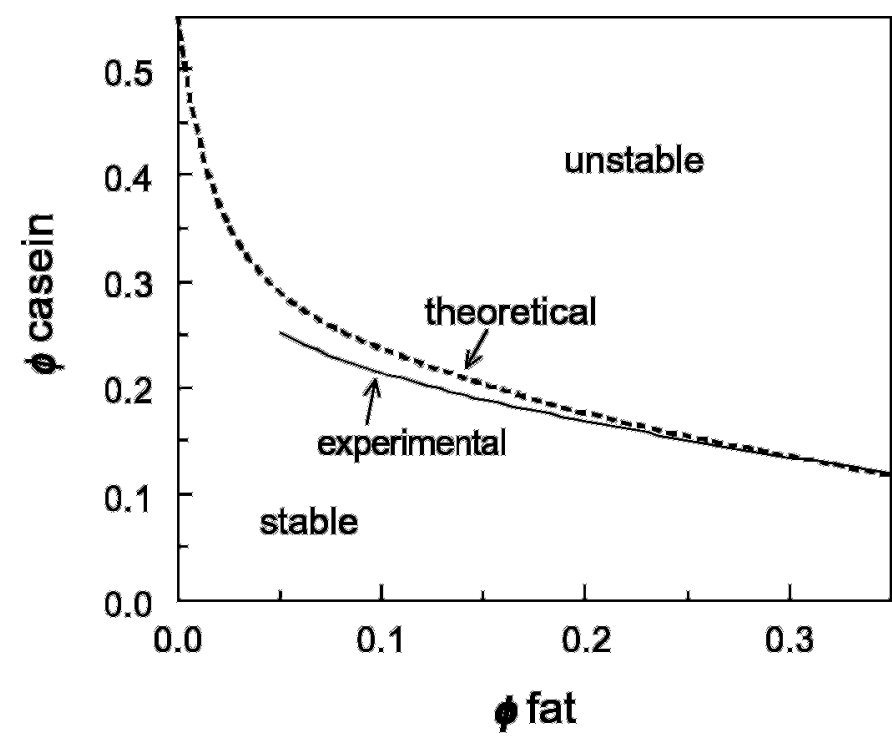

Figure 8. Experimental phase boundary compared with theoretical phase boundary.

imentally determined phase boundary. A close match is found between theory and experiment, which is quite surprising, since the casein micelles were considered as fully penetrable spheres in this theory. In reality, the casein micelles will behave more as 'hard sphere' colloidal particles, although they are of course not solid entities, but contain considerable amounts of water and have a quite open structure Because of the more or less hard sphere behavior of the casein micelles, it can be expected that the phase boundary should be positioned at lower volume fractions of casein micelles. However, the theory also does not take the polydispersity of the colloidal particles into account. Both the casein micelles and the oil droplets are natural systems and are not uniform in size. The effect of the polydispersity on the position of the phase boundary is the inverse of the effect of the casein micelles being colloidal instead of polymeric. These effects might well be of the same order of magnitude and have a zero net result on the position of the phase boundary, but this was not further investigated.

The difference in the kinetics of the phase separation between samples containing high and low total volume fractions of casein micelles and oil droplets can be explained by the effect of the casein micelles and the oil droplets on the viscosity. The phase separation is slower for systems that contain higher volume fractions of both colloids. The effect that the position of the interface changes rapidly directly after mixing of the colloids, but slows down after several hours could be a result of the increase of the concentration of oil droplets in the oil phase during the phase separation process. As the 
effective volume fraction of oil droplets in the upper phase increases, it will become increasingly difficult for additional oil droplets to enter this phase. This filling of the upper and lower phase with colloidal particles can also be observed from the data in Figure 6. This figure can be explained by assuming that both the lower and upper phase consist of colloids that can reach a maximum "effective" volume fraction of ca. 0.65 . Phase separation can only occur when the sum of the volume fraction of oil droplets and the volume fraction of casein micelles does not exceed this number. If the added volume fractions exceed 0.65 , the casein micelles remain mixed with the oil droplets and a network of oil droplets filled by casein micelles will probably be formed. The maximum in the sum of the two-volume fractions is reflected in the extrapolation of the curves in Figure 6 to the point where the position of the interface is 0 .

In conclusion, it has been shown that depletion interaction between colloids can occur in systems composed of (model) milk components. The interactions between casein micelles and fat globules cannot be neglected in concentrated milk-based products and can be explained by existing theory. However, other aspects that are important for these systems such as the effect of milk fat crystallization and the effect of salts should always be kept in mind as well.

\section{REFERENCES}

Dalgleish, D. G. 1997. Adsorption of protein and the stability of emulsions. Trends Food Sci. Technol. 8:1-6.

Dalgleish, D. G. 1998. Casein micelles as colloids: Surface structures and stabilities. J. Dairy Sci. 81:3013-3018.
De Kruif, C. G. 1999. Casein micelle interactions, Int. Dairy J. 9:183-188.

Dickinson, E. 1997. Properties of emulsions stabilized with milk proteins: overview of some recent developments. J. Dairy Sci. 80:2607-2619.

Dickinson, E., and M. Golding. 1997. Depletion flocculation of emulsions containing unadsorbed sodium caseinate. Food Hydrocolloids 11:13-18.

Dinsmore, A. D., A. G. Yodh, and D. J. Pine. 1995. Phase diagrams of nearly hard-sphere binary colloids. Phys. Rev. E 52:4045-4057.

Holt, C. 1985. The size distribution of bovine casein micelles: A review. Food Microstruct. 4:1-10.

Holt, C., and D. S. Horne. 1996. The hairy casein micelle. Neth. Milk Dairy J. 50:85-111.

Horne, D. S. 1998. Casein interactions: casting light on the black boxes, the structure in dairy products. Int. Dairy J. 8:171-177.

Jenness, R., and J. Koops. 1962. Preparation and properties of a salt solution that simulates milk ultrafiltrate. Neth. Milk Dairy J. 16:153-164.

Jeurnink, T. J. M., and C. G. de Kruif. 1993. Changes in milk on heating: Viscosity measurements. J. Dairy Res. 60:139-150.

Mao, Y., M. E. Cates, and H. N. W. Lekkerkerker. 1995. Depletion force in colloidal systems. Physica A 222:10-24.

Poon, W. C. K. 1998. Phase separation, aggregation and gelation in colloid-polymer mixtures and related systems. Curr. Opin. Colloid Interface Sci. 3:593-599.

Robins, M. M. 2000. Emulsions-creaming phenomena. Curr. Opin. Coll. Interface Sci. 5:265-272.

Snoeren, T. H. M., H. J. Klok, A. C. M. van Hooydonk, and A. J. Damman. 1984. The voluminosity of casein micelles. Milchwissenschaft 39:461-463.

Tuinier, R., E. ten Grotenhuis, C. Holt, P. A. Timmins, and C. G. de Kruif. 1999. Depletion interaction of casein micelles and an exocellular polysaccharide. Phys. Rev. E. 60:848-856.

Vélez-Ruiz, J. F., and G. V. Barbosa-Cánovas. 2000. Flow and structural characteristics of concentrated milk. J. Texture Stud. 31:315-333.

Vrij, A. 1976. Polymers at interfaces and the interactions in colloidal dispersions. Pure Appl. Chem. 48:471-483.

Walstra, P., and R. Jenness. 1984. Dairy Chemstry and Physics. John Wiley and Sons, New York.

Xu, W., A. Nikolov, D. T. Wasan, A. Gonsalves, and R. P. Borwankar. 1998. Fat particle structure and stability of food emulsions. J. Food Sci. 63:183-188. 